\title{
Comparison Analysis of Atmospheric Methane Absorption Cross Section
}

\author{
Liao Xiuying \\ 1. Institute of Geographic Sciences and Natural Resources Research, Chinese Academy of Sciences, Beijing,China \\ 2. Hunan University of Science and Technology, Graduate University of Chinese Academy of Sciences, Xiangtan, P.R. \\ China \\ 3. Graduate University of Chinese Academy of Sciences, Beijing, P.R. China
}

\begin{abstract}
Methane is an important anthropogenic greenhouse gas and is increasing steadily since the industrial revolution. SCIAMACHY on ENVISAT is the first satellite instrument whose measurements are sensitive to concentration changes of methane gas at all altitude levels down to the Earth's surface where the source/sink signals are largest. To retrieve the methane column amount from SCIAMACHY data, the methane absorption cross section is the prerequisite reference parameter. We based on theoretical calculation and calculate the methane cross section with HITRAN2004 and HITRAN2008 respectively. The results showed that there exits huge difference between the two cross sections. In order to improve the retrieval accuracy of methane column amount, we come to conclude that HITRAN2008 is the better choice for future calculation research.
\end{abstract}

Keywords-Methane, cross section, HITRAN, Comparison analysis

\section{INTRODUCTION}

We used SCIAMACHY data, which is part of the payload of ESA's Environmental Satellite (ENVISAT), and was launched into a sun-synchronous orbit on the 1st March 2002. It is a UV/visible/NIR (240-2380 nm) grating spectrometer that observes the upwelling radiance from the earth's atmosphere/surface and the extraterrestrial solar irradiance [1]. No matter what method we use in the inversion, we are required to obtain a variety of atmospheric absorption cross section, then to do fit between satellite observations spectrum and atmospheric radiative transfer model spectrum with corresponding optimization theory. How to get high-precision atmospheric methane absorption cross section becomes the necessary requirement for later work.

Using optical remote sensing data to retrieve greenhouse gas methane, its absorption spectrum is the prerequisite reference line. We generally use HITRAN spectral database, which contains the spectral parameter data of 39 basic atmospheric molecules and ions. On the demand of current research, we have to get the methane column amount from optical remote sensing data, so methane absorption crosssection is an essential parameter in the inversion process.

\section{HITRAN}

The HITRAN database is the recognized international standard, used for a vast array of applications including terrestrial and planetary atmospheric remote sensing, transmission simulations, fundamental laboratory spectroscopy studies, industrial process monitoring, and pollution regulatory studies. An international HITRAN advisory committee, composed of a dozen experts in the field of spectroscopy, has been established under the auspices of NASA. This committee reviews and evaluates new data and makes recommendations for updates and replacements in the compilation [2].

Many recent developments have pushed the requirements of HITRAN in terms of accuracy and degree of completeness. Among these developments one can cite the retrievals that various satellite remote-sensing missions are now capable of due in part to the high signal-to-noise ratio of the spectra and to advances in retrieval algorithms. Notable satellite spectrometer instrumentation includes MLS (Microwave Limb Sounder) [3] and TES (Tropospheric Emission Spectrometer) [4] on the Aura platform, and SCIAMACHY (Scanning Imaging Absorption spectrometer for Atmospheric chartography) on ENVISAT, ACE-FTS (Atmospheric Chemistry Experiment) [5] on SCISAT, AIRS (Atmospheric Infrared Sounder) [6] on Aqua, and GOSAT (Greenhouse gases Observing Satellite) [7]. These satellite instruments have put demands on HITRAN that include increased accuracy (by almost an order of magnitude in some cases) for the basic parameters: line position in vacuum wave number, $\mathrm{n}$ (in $\mathrm{cm} 1$ ), intensity of the line, $\mathrm{S}$ (in cm_1/(moleculecm_2) $)$, and line-shape parameters. 3 They also require more species, additional molecular bands, and weak lines throughout the spectral region covered by HITRAN (microwave through UV). In fact, the remotesensing experiments have demonstrated that the basic Lorentz line-shape parameter for Collisional broadening used in HITRAN, from which it is possible to calculate the Voigt line profile, is not satisfactory in many cases. To reduce the residuals between observation and simulation, it has often been necessary to invoke more sophisticated nonVoigt line shape functions such as Rautianor Galatry and line mixing.

\section{THEORETICAL CALCULATION AND IMPLEMENTATION OF ABSORPTION CROSS SECTION}

Linear molecules such as methane and other gases, the absorption cross section $\sigma$ is temperature and pressure dependent, and it can be expressed in wave number $v$ as Eq.(1) [8]:

$$
\sigma\left(v-v_{0}\right)=S(T) f\left(v-v_{0}\right)
$$


Where $S(T)$ is temperature $T$ dependent line intensity, $f$ is line function, $v_{0}$ is center position of the absorption line. Generally in the selected remote sensing satellite sensor channels (wavelength or wave number) range fingerprints, will contain a variety of gases absorption cross section, so we must consider the impact of these gases. Line intensity at temperature $T$ can be calculated with intensity of the reference temperature ${ }^{T_{0}}$ as Eq.(2) shows:

$$
S(T)=S_{0} \frac{Q_{0}^{v}}{Q^{v}(T)} \frac{Q_{0}^{r}}{Q^{r}(T)} \exp \left[\frac{E_{I}}{\kappa}\left(\frac{1}{T_{0}}-\frac{1}{T}\right)\right]
$$

Simulated emitted

term $\left([1-\exp (-h c v / \kappa T)] /\left[1-\exp \left(-h c v / \kappa T_{0}\right)\right]\right)$ must be unity. Here we ignored this term ( for SCIAMACHY, $v$ is more than $4000 \mathrm{~cm}^{-1}(<2400 \mathrm{~nm})$, and $\kappa T$ corresponds to about more than $200 \mathrm{~cm}^{-1}$ and atmospheric temperature). The term $\left(Q_{0}^{v} / Q^{v}\right)\left(Q_{0}^{r} / Q^{r}\right)$ contains the ratio of Vibration function and rotational function at reference temperature ${ }^{T_{0}}$ and actual temperature $T$.

In the usual experimental conditions, line shape of the linear function is mainly composed of the Gauss linear Doppler broadening and pressure broadening Lorrentz line shape, and is the convolution of the two, that Voigt linear function, as Eq.(3) shows:

$$
f_{v}\left(v_{0}-v\right)=\int_{-\infty}^{+\infty} \frac{\gamma^{L}}{\pi} \sqrt{\frac{\bar{m}}{2 \pi \kappa T}} \frac{1}{\left(v-v_{0}-\frac{u v_{0}}{c}\right)^{2}+\left(\gamma^{L}\right)^{2}} d u
$$

$m$ is the molecular mass of the interested molecular,

which is come from another file. And $\gamma_{L}$ can be expressed in Eq.(4):

$$
\gamma_{L}=\gamma_{L 0}\left(p / p_{0}\right)\left(T_{0} / T\right)^{n}
$$

To calculate implementation we need do some adjust to Voigt functions, as Eq.(5):

$$
\begin{aligned}
f_{G}(v) & =\frac{1}{\sigma \sqrt{2 \pi}} \exp \left(-\frac{\left(v-v_{0}\right)^{2}}{2 \sigma^{2}}\right) \\
\gamma_{G} & =\sqrt{8 \ln 2} \sigma \\
f_{L}(v) & =\frac{2 \gamma_{L} / \pi}{\gamma_{L}^{2}+4\left(v-v_{0}\right)^{2}} \\
f_{V} & =f_{G} \otimes f_{L} \\
& =\frac{2}{\gamma_{G}} \sqrt{\frac{\ln 2}{\pi}} V(X, Y)
\end{aligned}
$$

And $f_{G}, f_{L}, f_{V}$ is Gauss, Lorrentz and Voigt line function respectively, and $\gamma_{G}, \gamma_{L}$ are full width at half maximum(FWHM) of Gauss and Lorrentz line. $\sigma$ is variance of Gauss function, $V(X, Y)$ is Voigt function as Eq.(6) shows:

$$
\begin{aligned}
V(X, Y) & =\frac{Y}{\pi} \int_{--\infty}^{+\infty} \frac{e^{-z^{2}}}{Y^{2}+(X-z)^{2}} d z \\
X & =\frac{2 \sqrt{\ln 2}}{\gamma_{G}}\left(v-v_{0}\right) \\
Y & =\frac{\gamma_{L}}{\gamma_{G}} \sqrt{\ln 2}
\end{aligned}
$$

And Gauss line width $\gamma_{a}$ can be theoretical Doppler broadening width in Eq.(7):

$$
\gamma_{G}=7.16 \times 10^{-7} \sqrt{T / M v}
$$

Where $\mathrm{T}, \mathrm{M}, v$ are temperature $(\mathrm{K})$, molecular mass and line position $\left(\mathrm{cm}^{-1}\right)$ respectively. There is an important difficulty during calculation is that there is no simple analytical function form for Voigt function. If we calculate with the integral directly, there will be very large computational amount. Here we adopt the approximation forms to calculate the Voigt function as Eq.(8):

$$
V(X, Y)=\sum \frac{C_{i}\left(Y-A_{i}\right)+D_{i}\left(X-B_{i}\right)}{\left(Y-A_{i}\right)^{2}+\left(X-B_{i}\right)^{2}}
$$

Including $A_{i} \rightarrow D_{i} 16$ parameters and are listed in form 1 , compared to linear combination of Gauss approximation and Lorrentz approximation, this method has higher precision. And the biggest difference between calculated value and approximation Voigt value is less than $5 \times 10^{-4}$ (suppose the center height of function is unity). Another advantage of this method is that there are no additional parameters.

TABLE I. 16 PARAMTERS OF VOIGT FUNCTION APPROXIMATION METHOD

\begin{tabular}{ccccc}
\hline & $\mathbf{A}$ & $\mathbf{B}$ & $\mathbf{C}$ & $\mathbf{D}$ \\
\hline 1 & -1.2150 & 1.2359 & -0.3085 & 0.0210 \\
2 & -1.3509 & 0.3786 & 0.5906 & -1.1858 \\
3 & -1.2150 & -1.2359 & -0.3085 & -0.0210 \\
4 & -1.3509 & -0.3786 & 0.5906 & 1.1858 \\
\hline In & our & practical application in addition the & choice of
\end{tabular}

In our practical application, in addition the choice of theoretical method, we have to take the calculation cost into account. Based on forementioned theory, we have developed the procedure with matlab program language and related parameters from HITRAN2004 and HITRAN2008 respectively. During the inversion, we chose the SCIAMACHY channel 6 , so in this study we just did comparison analysis in this wavelength interval. Suppose $\mathrm{P}=900 \mathrm{hPa}, \mathrm{T}=280 \mathrm{~K}$, and the wave number interval is 0.01 $\mathrm{cm}-1$ in the calculation. 


\section{COMPARISON RESUlTS}

Here we choose a very small fingerprint interval of channel 6 to do comparison analysis. With the HITRAN2004, the calculated methane cross section is as figure 1 shows. It shows that during this fingerprint interval, methane cross section changes with wavelength and this is the intrinsic characteristic. And the unit of cross section is $\mathrm{cm} 2 /$ molecule. With the HITRAN2008, the calculated methane cross section is as figure 2 shows. In the whole this is similar with the former one, but there exists differences. In order to comparison directly, here I define the difference ratio Dratio as follows:

$$
D_{\text {ratio }}=\frac{\sigma_{2004}-\sigma_{2008}}{\sigma_{2004}} \times 100 \%
$$

And the difference ratio showed in figure 3 and figure 4.

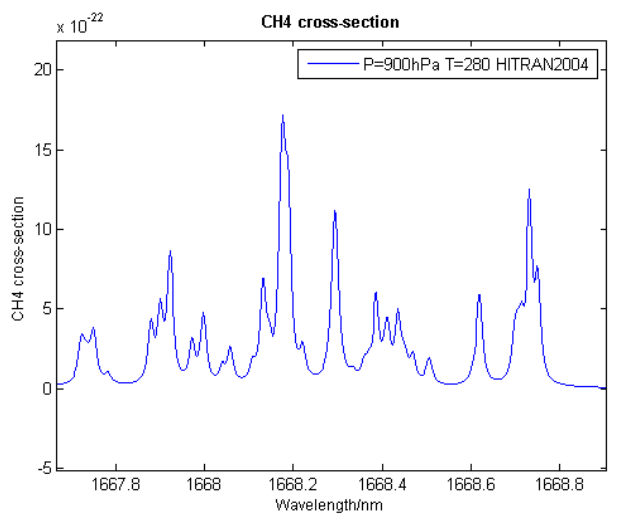

Figure 1. Methane absorption cross section retrieved with HITRAN2004

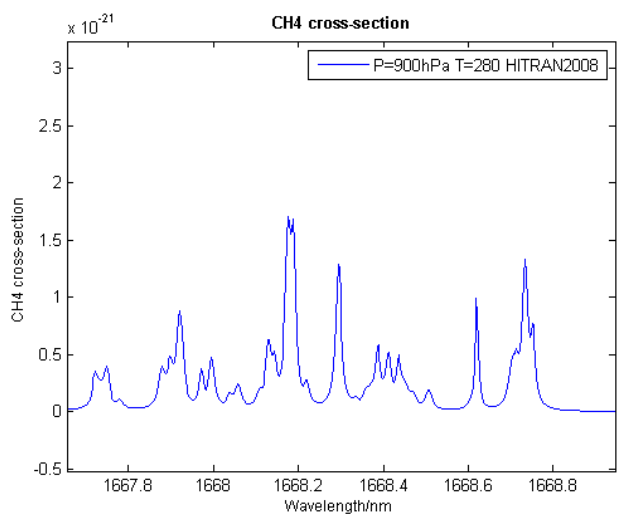

Figure 2. Methane absorption cross section retrieved with HITRAN2008

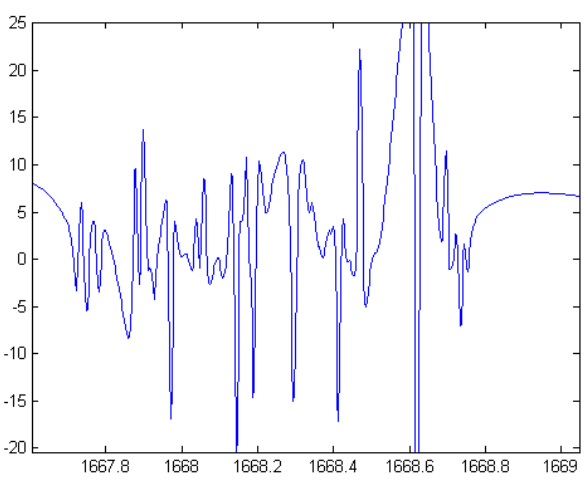

Figure 3. methane cross section difference ratio of different HITRAN

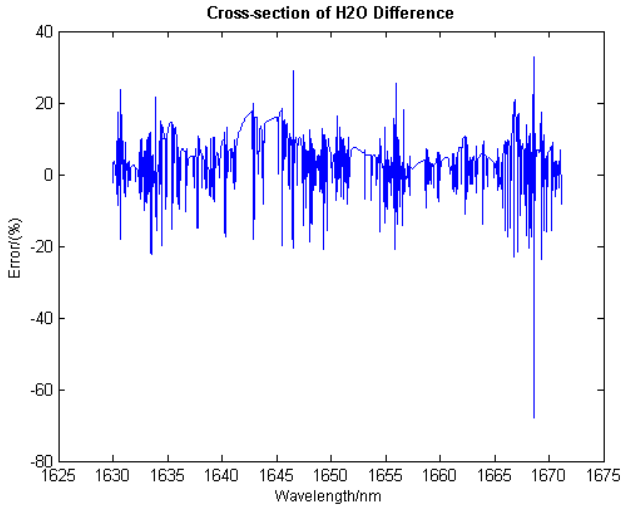

Figure 4. methane cross section difference ratio of different HITRAN during channel 6

\section{CONCLUSION}

During the atmospheric gas retrieval, different HITRAN database will bring huge errors in the retrieval accuracy because different HITRAN database exist huge differences for the gas absorption cross section. So we should make use of the new edition of HITRAN in our future research.

\section{ACKNOWLEDGMENT}

The research was funded by environmental protection public welfare special research - research on both the temporal and spatial patterns of greenhouse gas and its influences on climate effect (200909018). Special thanks to Pro. Sun Jiulin and other program members. Thanks to Ph.D Wang Zijun who provides some technological help for me.

\section{REFERENCES}

[1] Schneising, O., Analysis and interpretation of satellite measurements in the near-infrared spectral region: Atmospheric carbon dioxide and methane. Doctor Dissertation paper. University Bremen. 2008

[2] Rothman LS, Gordon I.E., Barbe A, Chris Benner D, et al. The HITRAN 2008 molecular spectroscopic database. JQSRT 2009.

[3] Waters JW, Froidevaux L, Harwood RS, Jarnot RF, Pickett HM, ReadWG, et al. The earth observing system microwave limb sounder (EOS MLS) on the Aura Satellite. IEEE Trans Geosci Remote Sensing 2006;44:1075-92. 
[4] Beer R. TES on the aura mission: scientific objectives, measurements, and analysis overview. IEEE Trans Geosci Remote Sensing 2006;44:1102-5.

[5] Bernath PF, McElroy CT, Abrams MC, Boone CD, Butler M, CamyPeyret $\mathrm{C}$, et al. Atmospheric chemistry experiment (ACE): mission overview. Geophys Res Lett 2005;32:5.

[6] Aumann HH, Chahine MT, Gautier C, Goldberg MD, Kalnay E, McMillin LM, et al. AIRS/AMSU/HSB on the aqua mission: design, science objectives, data products, and processing systems. IEEE Trans Geosci Remote Sensing 2003;41:253-64.

[7] Yokomizo M. Greenhouse gases observing SATellite (GOSAT) ground systems. Fujitsu Sci Techn J 2008;44:410-7.

[8] Le Wang. IR Absorption Spectroscopy Studies of Atmospheric Interested Molecules. Doctor Dissertation paper. University of Science and Technology of China. 2006 